\title{
Immune disorders in anorexia
}

\author{
SYLWIA MAEGORZATA SEOTWIŃSKA ${ }^{1}$, ROBERT SŁOTWIŃSKI ${ }^{2,3}$
}

\author{
${ }^{1}$ Department of Conservative Dentistry, Medical University of Warsaw, Poland \\ ${ }^{2}$ Department of Immunology, Biochemistry and Nutrition, Medical University of Warsaw, Poland \\ ${ }^{3}$ Department of Surgical Research and Transplantology, Mossakowski Medical Research Centre, Polish Academy of Sciences, Warsaw, \\ Poland
}

\begin{abstract}
Anorexia nervosa is a disease involving eating disorders. It mainly affects young people, especially teenage women. The disease is often latent and occurs in many sub-clinical and partial forms. Approximately from $0.3 \%$ to $1 \%$ of the population suffers from anorexia. It has been shown that patients with anorexia develop neurotransmitter-related disorders, leading to uncontrolled changes in the immune and endocrine systems. Interactions between cytokines, neuropeptides, and neurotransmitters play an important role in disease development. Significant malnutrition induces disorders and alterations in $T$-cell populations. The cellular response in patients with anorexia nervosa has been shown to be normal, although opinions on this issue are controversial. Laboratory studies on neutrophils in anorexia patients showed decreased adhesion and reduced bactericidal and cell activities. Despite such unfavourable results, patients with anorexia are resistant to infections, which are very rare in this group. Glutamine improves the performance of the human immune system. The administration of glutamine to anorexia patients, as a supplement to parenteral nutrition, has resulted in significant improvements in immune system parameters. The results of previous studies on the causes and risk factors in the development of anorexia nervosa are still ambiguous. One can hope that the differences and similarities between patients with anorexia nervosa and those with other forms of protein-calorie malnutrition may be helpful in determining the relationship between nutritional status and body defences and susceptibility to infection, and can help to broaden the knowledge about the aetiopathogenesis of anorexia nervosa.
\end{abstract}

Key words: anorexia nervosa, aetiology, immune disorders, treatment.

(Centr Eur Immunol 2017; 42 (2): 294-300)

Anorexia nervosa (AN) is a mental illness characterised by nutritional disorders, which has been a very serious and increasing problem for many years for many medical professionals of various specialties [1-3]. This disease mainly affects young people, especially teenage women. Precise determination of the incidence of this pathology is difficult because the disease is often latent and occurs in many subclinical and partial forms. It is believed that anorexia affects from $0.3 \%$ to $1 \%$ of the population, depending on the criteria applied [4-6]. The primary goal of physicians is to limit the development of this condition through early diagnosis and proper therapy aimed at a complete cure of this highly insidious disease.

Patients with eating disorders have great problems with proper interpersonal relationships. The appropriate protocol for medical and psychological treatment is essential in establishing a dialogue with the sick person, which requires knowledge, commitment, and patience from the medical team. This is of paramount importance in the clinical diagnosis and plays a decisive role in the therapeutic process and prognostic elements.
Scientists agree that starvation and malnutrition as well as any single nutritional deficiencies are all important causes of impaired functioning of the human immune system and are primarily responsible for significant decreases in cellular immunity [7-9].

During the course of anorexia, there are metabolic changes that can lead to serious biochemical disorders, resulting in dysfunction of many organs and systems. The occurrence of pathological conditions within the immune system is particularly dangerous and can cause a decrease in organism immunity. Immunological aspects of AN are the subject of many studies.

On the other hand, it should also be noted that the functioning of the immune system is controlled by the neuroendocrine system. It has been shown that patients with anorexia are very likely to experience severe hormonal imbalances, particularly with regard to secretion of sex hormones and cortisol, and disorders associated with the production of various neurotransmitters, resulting in uncontrolled changes in the immune system. Numerous works emphasised the importance of immunoendocrine

Correspondence: Sylwia Słotwińska, Department of Conservative Dentistry, Medical University of Warsaw, Miodowa 18, 00-246 Warszawa, Poland, e-mail: sylwia.slotwinska@wum.edu.pl

Submitted: 29.03.2017; Accepted: 7.04.2017 
factors in anorexia aetiopathogenesis. It is broadly discussed that interactions between cytokines, neuropeptides, and neurotransmitters play an important role in the course of AN. Brambilla et al. have shown a significant reduction in cholecystokinin-8 (CCK-8) and a significant increase in $\beta$-endorphin in patients with anorexia compared to healthy subjects [10]. Changes in the immune system of people with anorexia are atypical. The interaction between the immune system and the neuroendocrine network is very often emphasised. Many studies have demonstrated functional interdependence of the nervous and immune systems. Holden et al. have presented a very interesting concept, whereby the interaction between cytokines, neuropeptides, and neurotransmitters is the decisive factor in anorexia aetiopathogenesis. The resulting cascade of events leads to significant weight loss with concomitant increase in tumour necrosis factor $\alpha(\mathrm{TNF}-\alpha)$ and a decrease of interleukin 2 (IL-2) and 4 (IL-4) levels, which in turn induces the elevation of norepinephrine (noradrenaline) and a decrease in serotonin levels, important neurohormones and neurotransmitters [11].

Cytokines play one of the major functions in systemic metabolism regulation. They also play an important role in the control of infections and inflammatory reactions and modulate tissue and neuronal destruction. Cytokine synthesis is induced under a variety of physiological conditions, including hormonal changes, such as during menstrual cycle or after exercise. There are also strong interactions between the cytokines and the central nervous system, which can lead to a decrease in cytokine production by the immune system. Therefore, it is believed that cytokines, especially proinflammatory cytokines, may be a key component in the development of immunodeficiency in AN patients.

Corcos et al. evaluated a wide array of cytokines [IL-1, IL-2, IL-4, IL-6, IL-10, interferon $\gamma$ (IFN- $\gamma$ ), TNF- $\alpha$, and TGF- $\beta 2$ ] in AN patients. However, significant differences were observed only in the case of IL- 2 and TGF- $\beta 2$ in comparison with the control group [12]. Nagata et al. evaluated the lymphocyte proliferation response after mitogen phytohaemagglutinin (PHA) stimulation and investigated cytokine secretion ability in 17 anorexia patients. The results of this study suggest that the ability to produce cytokines may be associated with the neuroendocrine system and autonomic nervous system, which requires thorough studies and observations in a larger population [13].

Another study conducted in anorexia patients reported significant increases in TNF- $\alpha$ and markers of the socalled oxidative stress - oxygen shock (selenium, retinol, glutathione, ascorbic acid, $\alpha$-tocopherol, and peroxidase) compared to control subjects. In addition, a correlation was demonstrated between the assessed parameters and body mass index (BMI), which may suggest a significant association of these factors with anorexia development and progression [14]. A study analysing body mass index (BMI) in subjects with anorexia in the context of other parameters showed that significant malnutrition induced disorders and changes in T-cell populations. Saito et al. found a positive correlation between total T-cell pool and BMI, and a negative correlation between BMI and CD4 cells [15].

Protein-energy malnutrition (PEM), also referred to as protein-calorie malnutrition, is the most common cause of human immunodeficiency. PEM is not limited to developing countries; it is also equally often diagnosed in hospitalised patients in developed countries. Reduced cellular immunity is the most common immune deficiency in PEM with reduced in vivo response in late-type hypersensitivity tests and reduced lymphocyte transformation responses to mitogens in vitro. However, these abnormalities significantly improve after re-nutrition. A decrease in the number of peripheral blood serum T-cells is one of the characteristic symptoms of protein-energy malnutrition, which usually leads to functional abnormalities. Infections that impair both nutrition and the immune system are frequent complications in PEM. It is therefore difficult to conclusively determine which factors in PEM are responsible for changes and disorders in cellular immunity $[16,17]$.

Patients with AN are a relatively homogeneous population, within which a significant energy deficit is observed in individual subjects. Studies have shown that their diet is low in carbohydrates, while protein and fat intake is maintained at an acceptable level. Despite significant malnutrition, or even starvation, patients with anorexia are resistant to infections, which are very rare in this group. The results of numerous studies have shown that the cellular response in patients with $\mathrm{AN}$ is normal, although opinions on this issue are controversial. Researchers at the University of Semmelweis in Budapest did not reveal significant differences in the range of immunological parameters assessed in anorexia subjects compared to healthy subjects, and did not confirm the relationship between the immune status and the clinical status of patients with anorexia [18-20]. Similar results were obtained by Komorowska-Pietrzykowska et $\mathrm{al}$., who examined 29 young women, aged 12-20 years, with AN diagnosis, who did not manifest any significant disorders in immune system parameters; only a slight tendency of lower CD4/CD8 cell ratio and low CD3 cell counts were found [21].

Many authors believe that AN is an unusual form of protein-calorie malnutrition characterised by normal values of T-cell counts and a significant reduction in the percentage of CD8 cells compared to other forms of protein-calorie malnutrition, where significant deficiencies are observed in the T-cell subpopulation. Severe protein-calorie malnutrition syndromes are characterised by a very high sensitivity to all infections and a greatly impaired immune response. An increase in the $\mathrm{CD} 4 / \mathrm{CD} 8$ ratio and higher CD20 cell count are often found in such patients. A markedly significant decrease in the percentage of CD4 cells is a particularly characteristic feature [22]. This is not ob- 
served in AN patients because CD4 values are normal [23, 24]. In contrast, Nagata et al., similarly to other authors, demonstrated a significant increase in the percentage of CD4+ lymphocytes and a significant increase in the CD4/ $\mathrm{CD} 8$ ratio, and a significant reduction in sIL-2R soluble receptor concentration in peripheral blood serum in anorexia patients, compared to controls [25]. Bessler et al. conducted in vitro studies on the ability of peripheral blood mononuclear cells to produce IL-1, IL-2, IL-3. These authors pondered why, despite significant impairment of this function compared to healthy individuals, AN patients do not show significant differences in the incidence of typical infections. Perhaps the compensatory mechanism of the presence of additional agents in peripheral blood serum in AN patients, which stimulate cell interleukin secretion, can explain this phenomenon [26].

Brambilla et al. and Solmi et al. conducted very interesting studies on peripheral blood concentrations of selected proinflammatory cytokines: IL- $1 \beta$, IL-6, TNF- $\alpha$, IL-6 R, sIL-6-R, sTNF-alpha-RI, sTNF- $\alpha$-RII, and s-IL- $1 \beta$-R-A in anorexia patients. Concentration analysis did not show significant differences in the results obtained in the study group for most of the evaluated cytokines compared to the control group. Only the concentrations of interleukin 6 receptors (sIL-6-R, IL-6R) were found to be significantly reduced in AN patients compared to the control group [27, 28]. Pisetsky et al. and Kahl et al. performed similar studies showing a statistically significant increase in the peripheral blood levels of IL-6 in AN patients compared with the control group, which could indicate the involvement of this cytokine in the pathogenesis of anorexia nervosa $[29,30]$.

Anorexia patients very often have significantly reduced late-type hypersensitivity response, which is typically observed in PEM in chronically malnourished, starving children in Third World countries, and in patients hospitalised for severe malnutrition accompanying devastating chronic diseases. The main reason is markedly reduced T-cell counts in peripheral blood serum, especially T-helper cells, and to a lesser extent suppressor T-lymphocytes. Studies on immunocompetent cells in patients with anorexia and bulimia nervosa showed statistically significant reductions in leukocyte and lymphocyte values as well as CD2 and CD4 cells. The authors of this work explained this fact by the significant dominance of the neuroimmunological network determinants over harmful factors resulting from malnutrition. It was also established that the so-called "naive cells" are particularly susceptible to malnutrition [31,32].

Tumour necrosis factor is a cytokine that is involved in various metabolic processes. Cytokines can directly interact with hunger centres, especially IL-1 and TNF, which additionally affects peripheral signals to satiety centres, leading to temporary gastric emptying inhibition. Particularly interesting is the ability to mediate weight loss mechanisms by central inhibition of food intake and lipoprotein lipase blockade, and catabolic effects on adipose tissue, which have been very well documented in both in vitro studies and experimental studies in animals. It is known that acute starvation, induced by human peripheral blood mononuclear cells (PBMCs), results in significantly increased production of TNF in vitro.

Peripheral blood mononuclear cells have been studied in patients with anorexia; TNF production, IFN activity, cellular cytotoxicity (CMC), and the ratio of these parameters to plasma levels of $\beta$-endorphin were evaluated. The group of anorexia subjects had significantly reduced cellular cytotoxicity and markedly increased spontaneous TNF production in vitro; however, there were no significant differences in plasma $\beta$-endorphin levels and interferon system activity compared with control subjects. The mechanisms causing the described changes remain unclear. Increased TNF production has a significantly adverse effect on anorexia because it stimulates an exacerbating central effect of food intake suppression and/or increased tissue catabolism [33].

Many authors believe that immune deficiencies are secondary processes to malnutrition, which is observed in the development and progression of anorexia. Hence, the view that the correct nutrition treatment should be the most appropriate standard for therapeutic treatment of anorexia. Numerous studies have been undertaken to assess the effects of starvation on immune system functioning, with particular regard to shifts in lymphocyte subpopulations. It has been shown that malnutrition causes a significant reduction in the percentage of T-cells. Mustafa et al. evaluated T-cell subpopulations prior to treatment and after the application of nutritional therapy in 20 anorexia patients. The results of these studies have shown that adequate dietary treatment may lead to a significant increase in total $\mathrm{T}$-cell counts and an increase in the percentage of CD8 cells compared to baseline [34]. A study by Schattner et al. demonstrated that nutrition treatment and dietetic rehabilitation in individuals with anorexia may result in both regulation of the immune response and a significant normalisation of cytokine secretion, as well as recovery of the assessed resistance parameters to values comparable to control groups $[35,36]$.

Allende et al. conducted a study in a group of 40 patients (DSM-IV-R) and showed that the immune system of anorexia patients was characterised by high instability, which can result from chronic and severe malnutrition. Deregulation of the immune system seems to start with a spontaneous and uncontrolled increase of the IL-1 $\beta$ and TNF- $\alpha$, which results in impaired balance and stimulation in the T-cell subpopulation and consequently changes in B-cell activation and function. As a result of intensive nutrition therapy, there was an overall tendency for normalisation in the immune system parameters and the gradual reversion of pre-existing disorders and immunodeficiencies in selected cases [37]. 
This fact was not confirmed by Nova et al., who studied the ability of peripheral blood mononuclear cells (PMN) to produce cytokines in 40 women with anorexia. The results of this study showed that TNF- $\alpha$ and IL-6 were reduced and IL-1 was elevated in patients with anorexia nervosa, compared to controls (35 subjects). However, after an intensive four-week treatment, no changes in cytokine concentrations were observed [38]. It may be necessary to widen the panel of investigated parameters by neuroendocrine factors and analyse the possibilities and immune capabilities of persons with anorexia in this context [39].

The same centre has come up with very interesting research on the usefulness of probiotic formulations in the nutritional treatment of anorexia nervosa patients. After 10 weeks of the nutritional treatment with the addition of probiotic yoghurt (Lactobacillus bulgaricus), favourable changes in immune system parameters were observed, i.e. a significant increase in IFN- $\gamma$ and a significant increase in the CD4+/CD8+ cell ratio [40].

The importance of supplementing the therapeutic diet with nutrients and high-energy components in the treatment of anorexia has been highlighted for a long time. Selected amino acids are particularly important and essential components for the proper development, growth, and function of immunocompetent cells, especially for lymphocytes and macrophages. A particular role is attributed to glutamine. Many works have shown that glutamine improves the functioning of the human immune system, particularly in critically ill patients. The administration of glutamine, as a supplement to parenteral nutrition, to anorexia patients has resulted in significant improvements in selected parameters evaluating the immune system status. After just 10 days of dietary therapy, a significant increase was achieved in neopterin peripheral blood serum concentration ( $26.44 \pm 3.08$ vs. $6.75 \pm 1.73 \mathrm{nmol} / 1, p<0.001$ ), and after 20 days of observation, a significant increase was seen in lymphocyte counts compared to the reference group that was also treated with parenteral nutrition, but without glutamine supplementation [41].

Despite many positive examples in the therapy of anorexia, it is still a very difficult disease to treat. Many patients require prolonged hospitalisation and constant monitoring of important diagnostic parameters, which is not always useful in assessing the degree of malnutrition and adequate to the clinical status of the patient. This applies, for example, to testing the albumin levels, which are one of the basic markers of assessing the nutritional status of the body. Serum albumin levels in peripheral blood in anorexia patients are very often normal, even in severe and advanced cases [42].

For many years, studies have been carried out in different research centres, which take into account the multifactorial anorexia aetiology. Numerous studies have demonstrated elevated levels of multiple chemokines and cytokines in the peripheral blood serum of anorexia nervosa patients. Raymond et al. demonstrated an increase in interferon gamma (IFN-gamma) secretion by mononuclear cells in vitro after concanavalin-A stimulation in anorexia nervosa patients compared to control [43].

Interesting research concerns the evaluation of complement activation in the course of anorexia. It is believed that elevated concentrations of complement components may have a significant association with severe complications that occur in advanced refractory anorexia.

The complement system plays a key role in the acute antibacterial response, as a major component of innate immunity, primarily responsible for the quality of antigen response. Flierl et al. have shown that complement C3 may be a potential biomarker for severity and progression of anorexia nervosa [44].

Many authors raise the importance of humoral immunity in the pathogenesis of anorexia nervosa. Clinical observations and experimental studies have shown that both in humans and experimental animals there are antibodies that regulate the level of peptide hormones and neuropeptides responsible for hunger and satiety [45].

People with anorexia have elevated levels of ghrelin, a hormone that is involved together with leptin and insulin in hunger control processes. Terashi et al. examined the occurrence of $\operatorname{IgG}, \operatorname{IgM}$, and $\operatorname{IgA}$ ghrelin antibodies and found that the level of these antibodies was significantly lower in anorexia patients [46].

Various aetiological and risk factors are considered that may be associated with the development and course of eating disorders, particularly in anorexia. A positive correlation has been found between smoking and the occurrence of mental illnesses, such as schizophrenia and bipolar affective disorder, as well as the course of bulimia and binge eating. However, at present, there is no clear evidence of such an association in the case of AN [47]. Very interesting research concerns the expression of adiponectin receptor genes in people with anorexia. There was no association found of this hormone with gender and BMI [48]. A significant decrease was observed in AN patients in an immunocompetent cell population with a parallel increase in antioxidative potential and higher anti-inflammatory parameters [49].

The presence of haematological disorders in anorexia patients is an additional factor that hampers the diagnostics and treatment in these individuals. There are numerous abnormalities in the composition and function of bone marrow during AN. In addition, many people with anorexia are diagnosed with red blood cell acanthocytosis, leukopaenia, complement damage and, above all, lower granulocyte activity [50]. Dietary deficiencies can decrease the number and phagocytic activity of polymorphonuclear granulocytes. It has been shown that the leukocyte's ability to eliminate bacterial antigens in AN patients is significantly reduced [51]. The neutrophil chemotactic abilities 
are impaired in severe malnutrition or in the course of infection. Neutrophils play an important bactericidal function. Any deficiencies and damage related to their activity or count are associated with an increased risk of bacterial infection. Laboratory studies of neutrophils in patients with anorexia showed decreased adhesion, reduced bactericidal capacity and decreased cell activity. Despite such unfavourable results, AN patients do not suffer from severe or chronic infectious diseases [52]. More than $80 \%$ of AN patients have anaemia (haematocrit $<37 \%$ ), $79 \%$ of AN patients have leukopaenia (WBC $<4.5 \mathrm{k} / \mu \mathrm{l}$ ), $29 \%$ have neutropaenia, $25 \%$ have thrombocytopaenia, and $17 \%$ of hospitalised patients develop thrombocytosis [53].

Recurrent bacterial infections are a common symptom of immunodeficiency diseases, including abnormal function of immunoglobulins, effector cells, and complement cells. Many people suffering from anorexia appear very healthy. In addition, although these patients exhibit immunodeficiencies and changes in immunological parameters, they do not suffer from viral infections, excluding cases of advanced weakness and malnutrition stages.

On the one hand, malnutrition has been epidemiologically and experimentally linked to increased susceptibility to infection, but on the other, an unexplained paradox remains: according to available studies, infections in AN are not common. Although isolated cases of pulmonary tuberculosis or cutaneous staphylococcal infections are sometimes observed in AN patients, so-called colds or flu are extremely rare [52, 54-56].

It is also worth stressing that both chronic stress and frequent depressive states that accompany people with anorexia certainly have a significant impact on the immune response of the organism. Therefore, many authors believe that immunosuppressive and/or immunomodulatory drugs may play an important role in the development of a new strategy for treating neurodegenerative diseases, including the improvement of therapeutic outcomes in anorexia patients [57, 58].

Cellular immunity parameters are those that clearly distinguish AN from other forms of protein-calorie malnutrition. A typical subpopulation profile in protein-calorie malnutrition is characterised by a significantly reduced $\mathrm{T}$-cell count and unchanged or slightly elevated B-cell numbers. These types of differences are usually very difficult to unequivocally distinguish between undernourished AN patients and control groups $[23,59,60]$.

Protein-calorie malnutrition, observed in extreme starvation, is related to the lack of carbohydrates, lipids, proteins, and vitamins and minerals, whereas in AN patients, very low carbohydrate intake is primarily observed, as compared to protein and lipid intake, which can be important in DNA synthesis and cell regeneration processes. Protein ingestion in AN is often at an appropriate or acceptable level, thus cell regeneration functions are usually preserved [61, 62].
The results of previous studies on the causes and risk factors in AN development are still ambiguous. One can hope that the differences and similarities between patients with AN and those with other forms of protein-calorie malnutrition may be helpful in determining the relationship between nutritional status and body defences and susceptibility to infection, and will help to broaden the knowledge about the aetiopathogenesis of AN.

The authors declare no conflict of interest.

\section{References}

1. American Psychiatric Association. Diagnostic and Statistical Manual of Mental Disorders, $4^{\text {th }}$ ed. Text Revision. Washington, DC: American Psychiatric Association; 1994.

2. World Health Organization. The ICD-10 classification of mental and behavioral disorders. Clinical description and diagnostic guidelines. Geneva: World Health Organization 1992.

3. Pritts S, Susman J (2003): Diagnosis of eating disorders in primary care. American Family Physician (Pro Quest Medical Library) 67: 297-308.

4. Fairburn CG, Harrison PJ (2003): Eating disorders. Lancet 361: 407-416.

5. Pull ChB (2004): Binge eating disorder. Curr Opin Psychiatry. Lippincott Williams \& Wilkins.

6. Hoek HW, Van Hoeken D (2003): Review of the prevalence and incidence of eating disorders. Int J Eat Disord 34: 383396.

7. Grimble RF (1998): Modification of inflammatory aspects of immune function by nutrients. Nutr Res 18: 1297-1317.

8. Pomposelli JJ, Flores EA, Bistrian BR (1988): Role of Biochemical Mediators in Clinical Nutrition and Surgical Metabolism. J Parenter Enteral Nutr 1988, 12: 212-18.

9. Chandra RK (2002): Effect of Post-natal Protein Malnutrition and Intrauterine Growth Retardation on Immunity and Risk of Infection pp 41-56. Nutrition and Immune Function. Edited by Calder PC, Field CJ, Gill HS. CAB International.

10. Brambilla F, Ferrari E, Brunetta M, et al. (1996): Immunoendocrine aspects of anorexia nervosa. Psychitry Res 62: 97104.

11. Holden RJ, Pakula IS (1996): The role of tumor necrosis factor-alpha in the pathogenesis of anorexia and bulimia nervosa, cancer cachexia and obesity. Med Hypotheses 47: 423-438.

12. Corcos M, Guilbaud O, Chaouat G, et al. (2001): Cytokines and anorexia nervosa. Psychosom Med 63: 502-504.

13. Nagata T, Tobitani W, Kiriike N, et al. (1999): Capacity to produce cytokines during weight restoration in patients with anorexia nervosa. Psychosom Med 61: 371-377.

14. Agnello E, Malfi G, Costantino AM, et al. (2012): Tumor necrosis factor alpha and oxidative stress as maintaining factors in the evolution of anorexia nervosa. Eat Weight Disord 17: 194-199.

15. Saito H, Nomura K, Hotta M, Takano K (2007): Malnutrition induces dissociated changes in lymphocyte count and subset proportion in patients with anorexia nervosa. Int J Eat Disord 40: $575-579$. 
16. Schaible UE, Kaufmann SHE (2007): Malnutrition and Infection: Complex Mechanisms and Global Impacts. PLoS Medicine 4: 806-812.

17. Tomkins A (2002): Nutrition, Infection and Immunity: Public Health Implications. Pp 375-412. Nutrition and Immune Function. Calder PC, Field CJ, Gill HS (eds.). CAB International.

18. Pali AA, Paszthy B (2008): Changes of the immune function in patients with eating disorders. Ideggyogy Sz 61: 381-384.

19. Paszthy B, Svec P, Tury F, et al. (2007): Impact of anorexia nervosa on activation characteristics of lymphocytes. Neuro Endocrinol Lett 28: 422-426.

20. Paszthy B, Svec P, Vasarhelyi B, et al. (2007): Investigation of regulatory T cells in anorexia nervosa. Eur J Clin Nutr 61: $1245-1249$

21. Komorowska-Pietrzykowska R, Rajewski A, Wiktorowicz K, Służewska A (1996): Immunological system activity in anorexia nervosa. Psychiatr Pol 30: 801-810.

22. Maltoni M, Fabbri L, Nanni O, et al. (1997): Serum levels of tumor necrosis factor alpha and other cytokines do not correlate with weight loss and anorexia in cancer patients. Support Care Cancer 5: 130-135.

23. Golla JA, Larson LA, Anderson CF, et al. (1981): An immunological assessment of patients with anorexia nervosa. Am J Clin Nutr 34: 2756-2762.

24. Fink S, Eckert E, Mitchell J, et al. (1996): T-lymphocytes subsets in patients with abnormal body weight: longitudinal studies in anorexia nervosa and obesity. Int J Eat Disord 20: 295-305.

25. Nagata T, Kiriike N, Tobitani W, et al. (1999): Lymphocyte subset, lymphocyte proliferative response, and soluble interleukin-2 receptor in anorexic patients. Biol Psychiatry 45: 471-474.

26. Bessler H, Karp L, Notti I, et al. (1993): Cytokine production in anorexia nervosa. Clin Neuropharmacol 16: 237-243.

27. Brambilla F, Monti D, Franceschi C (2001): Plasma concentrations of interleukin-1 beta, interleukin- 6 and tumor necrosis factor-alpha, and of their soluble receptors and receptor antagonist in anorexia nervosa. Psychitry Res 103: 107-114.

28. Solmi M, Veronese N, Favaro A, et al. (2015): Inflammatory cytokines and anorexia nervosa: a metaanalysis of crossectional and longitudinal studies. Psychoneuroendocrinology 51: 237-252.

29. Pisetsky DS, Trace SE, Brownley KA, et al. (2014): The expression of cytokines and chemokines in the blood of patients with severe weight loss from anorexia nervosa: an exploratory study. Cytokine 69: 110-115.

30. Kahl KG, Kruse N, Rieckmann P, Schmidt MH (2004): Cytokine mRNA expression patterns in the disease course of female adolescents with anorexia nervosa. Psychoneuroendocrinology 29: 13-20.

31. do Carmo I, Palma-Carlos ML, Melo A, et al. (1997): Characterization of leucocytes, lymphocytes and lymphocytes subsets in eating disorders. Allerg Immunol 29: 261-268.

32. Cason J, Ainley CC, Wolstencroft RA, et al. (1986): Cell-mediated immunity in anorexia nervosa. Clin Exp Immunol 64: 370-375.

33. Schattner A, Steinbock M, Tepper R, et al. (1990): Tumor necrosis factor production and cell-mediated immunity in anorexia nervosa. Clin Exp Immunol 79: 62-66.

34. Mustafa A, Ward A, Treasure J, Peakman M (1997): T lymphocyte subpopulations in anorexia nervosa and refeeding. Clin Immunol Immunopathol 82: 282-289.
35. Schattner A, Tepper R, Steinbock M, et al. (1990): TNF, interferon-gamma and cell-mediated cytotoxicity in anorexia nervosa. J Clin Lab Immunol 32: 183-184.

36. Schattner A, Tepper R, Steinbock M, et al. (1992): Cytokines in anorexia nervosa - nutritional or neuroimmunal changes. Harefuah 123: 245-247.

37. Allende M, Corell J, Manzanares J, et al. (1998): Immunodeficiency associated with anorexia nervosa is secondary and improves after refeeding. Immunology 94: 543-551).

38. Nova E, Gomez-Martinez S, Morande G, Marcos A (2002): Cytokine production by mononuclear cells from in-patients with anorexia nervosa. Br J Nutr 88: 183-188.

39. Nova E, Samartin S, Gomez, et al. (2002): The adaptive response of the immune system to the particular malnutrition of eating disorders. Eur J Clin Nutr 56 suppl 3: S34-37.

40. Nova E, Toro O, Varela P, et al. (2006): Effects of a nutritional intervention with yogurt on lymphocyte subsets and cytokine production capacity in anorexia nervosa patients. Eur J Nutr 45: 225-233).

41. Mondello S, Italiano D, Giacobbe MS, et al. (2010): Glutamine-supplemented total parenteral nutrition improves immunological status in anorectic patients. Nutrition 26: 677681.

42. Narayanan V, Gaudiani JL, Mehler PS (2009): Serum albumin levels may not correlate with weight status in severe anorexia nervosa. Eat Disord 17: 322-326.

43. Raymond NC, Dysken M, Bettin K, et al. (2000): Cytokine production in patients with anorexia nervosa, bulimia nervosa, and obesity. Int J Eat Disord 28: 293-302.

44. Flierl MA, Gaudiani JL, Sabel AL, et al. (2011): Complement $\mathrm{C} 3$ serum levels in anorexia nervosa: a potential biomarker for the severity of disease. Ann Gen Psychiatry doi: 10.1186/1744-859X-10-16.

45. Fetissov SO, Sinno MH, Coeffier M, et al. (2008): Autoantibodies against appetite-regulating peptide hormones and neuropeptides: Putative modulation by gut microflora. Nutrition 24: 348-359.

46. Terashi M, Asakawa A, Harada T, et al. (2011): Ghrelin reactive autoantibodies in restrictive anorexia nervosa. Nutrition 27: 407-413.

47. Solmi M, Veronese N, Sergi G, et al. (2016): The association between smoking prevalence and eating disorders: a systemic review and meta-analysis. Addiction doi: 10.1111/add.13457.

48. Alberti L, Gilardini L, Girola A, et al. (2007): Adiponectin receptors gene expression in lymphocytes of obese and anorectic patients. Diabetes Obes Metab 9: 344-349.

49. Omodei D, Pucino V, Labruna G, et al. (2015): Immune-metabolic profiling of anorexia patients reveals an antioxidant and anti-inflammatory phenotype. Metabolism 64: 396-405.

50. Kay J, Stricker RB (1983): Hematologic and immunologic abnormalities in anorexia nervosa. South Med J 76: 1008-1010.

51. Gotch FM, Spry CJF, Mowat AG, et al. (1975): Reversible granulocyte killing defect in anorexia nervosa. Clin Exp Immunol 21: 244-249.

52. Palmblad J, Fohlin L, Lundsrtom M (1977): Anorexia nervosa and polymorphonuclear (PMN) granulocyte reactions. Scand J Haematol 19: 334-342.

53. Sabel AL, Gaudiani JL, Statland B, Mehler PS (2013): Hematological abnormalities in severe anorexia nervosa. Ann Hematol 92: 605-613.

54. Bowers TK, Eckert E (1978): Leukopenia in anorexia nervosa: lack of increased risk of infection. Arch Intern Med 138: 1520-1523. 
55. Chandra RK, Newberne PM (1977): Nutrition, immunity, and infection: mechanisms of interactions. New York: Plenum Press.

56. Lucas AR (1977): On the meaning of laboratory values in anorexia nervosa. Mayo Clin Proc 52: 748-750.

57. Marcos A (2000): Eating disorders: a situation of malnutrition with peculiar changes in the immune system. Eur J Clin Nutr 54 Suppl 1: S61-4.

58. Berthold-Losleben M, Heitmann S, Himmerich H (2009): Anti-inflammatory drugs in psychiatry. Inflamm Allergy Drug Targets 8: 266-276.

59. Suskind RM (1977): Malnutrition and the immune response. New York: Raven Press.

60. Chandra RK (1974): Rosette-forming T lymphocytes and cell-mediated immunity in malnutrition. Br Med J 3: 608-609.

61. Chandra RK (1976): Nutrition as a critical determinant in susceptibility to infection. World Rev Nutr Diet 25: 166-188.

62. Sobotka L (2013): Podstawy Żywienia Klinicznego. Ed. 4 Krakowskie Wydawnictwo Scientifica Sp. z oo. 